\title{
Asymptotic Analysis for Optimal Investment and Consumption with Transaction Costs
}

\author{
Karel Janeček \\ Department of Mathematical Sciences \\ Carnegie Mellon University \\ Pittsburgh, PA 15213 \\ kjanecek@andrew.cmu.edu \\ Steven E. Shreve ${ }^{1}$ \\ Department of Mathematical Sciences and \\ Center for Computational Finance \\ Carnegie Mellon University \\ Pittsburgh, PA 15213 \\ shreve@cmu.edu
}

February 28, 2003

\begin{abstract}
We consider an agent who invests in a stock and a money market and consumes in order to maximize the utility of consumption over an infinite planning horizon in the presence of a proportional transaction $\operatorname{cost} \lambda>$. The utility function is of the form $U(c)=c^{1-p} /(1-p)$ for $p>0, p \neq 1$. We provide a heuristic and a rigorous derivation of the asymptotic expansion of the value function in powers of $\lambda^{1 / 3}$, and we also obtain asymptotic results on the boundary of the "no-trade" region.
\end{abstract}

Short title: Asymptotic Transaction Costs

JEL classification: G13

Mathematics Subject Classification (1991): 90A09, 60H30, 60G44

${ }^{1}$ Work supported by the National Science Foundation under grant DMS-01-01407. 


\section{Introduction}

We consider the problem of an agent seeking to optimally invest and consume in the presence of proportional transaction costs. The agent can invest in a stock, modeled as a geometric Brownian motion, and in a money market with constant rate of interest. She may also consume and get utility $U(c)=c^{1-p} /(1-p)$, where $p>0, p \neq 1$. In addition, the agent must pay a proportional transaction cost $\lambda>0$ for transferring capital between the stock and money market. All consumption is done from the money market. The agent wishes to maximize the expected discounted integral over $[0, \infty)$ of the utility of consumption.

When the transaction cost $\lambda$ is zero, the agent's optimal policy is to keep a constant proportion of wealth, which we call the Merton proportion and denote $\theta_{p}$, invested in the stock; see Merton [40]. When $\lambda>0$, the optimal policy is to trade as soon as the position is sufficiently far from the Merton proportion. More specifically, the agent's optimal policy is to maintain her position inside a wedge called the No Trade (NT) region. Trading occurs when the position hits the boundaries of the $N T$ region and no trading occurs in the interior of $N T$. If the agent's position is initially outside $N T$, she should immediately sell or buy stock in order to move to the boundary of NT. Except when the left boundary of $N T$ is the positive $y$-axis, the set of trading times has zero Lebesgue measure, and the total amount of capital traded can be characterized by a possible initial jump plus local time on the boundary of $N T$. When the left boundary of $N T$ is the positive $y$-axis, the agent will stay on the $y$-axis once she arrives there. This problem was formulated by Magill \& Constantinides [39], solved under restrictive conditions by Davis \& Norman [15], and thoroughly analyzed by Shreve \& Soner [43]. Although the nature of the solution can be characterized, there is no explicit formula for this solution.

The Hamilton-Jacobi-Bellman (HJB) equation for the optimal control problem with transaction costs is a partial differential equation in two variables. For the power utility functions considered here, this can be reduced to an equation in one variable. Numerical results are provided by [1], [46], [47]. A useful and perhaps more informative approach for obtaining explicit results, the approach of this paper, is to develop a power series expansion for the value function and the boundaries of the $N T$ region in powers of $\lambda$. For example, Constantinides [9] numerically computed the effect of transaction costs on the value function for our problem, and observed that transaction costs have a "first-order effect on assets' demand" and a "second-order effect on equilibrium asset return." In this paper, we make these statements precise by observing that the width of the NT region is large, of order $\lambda^{1 / 3}$, whereas the effect of transaction cost on the value function is smaller, of order $\lambda^{2 / 3}$.

The asymptotic expansion is valid for any Merton proportion $\theta_{p}$, except for the case in which all wealth is invested in stock $\left(\theta_{p}=1\right)$. In particular, we refute the conjecture in [43] that the Merton line (the set of stock/money market positions which are in the Merton proportion) is outside $N T$ for $\theta_{p}>1$. This is the case for sufficiently high transaction costs. However, for sufficiently small transaction costs the Merton line is inside NT. 
In the presence of transaction costs, contingent claim pricing by replication, or more generally by super-replication, has received considerable attention but often leads to a trivial result: the cheapest strategy is buy-and-hold. See [6], [14], [18], [20], [30], [31], [33], [34], [37], [44].

One alternative to the extremely conservative super-replication method for contingent claim prices, pioneered by Leland [36], is to strike a trade-off between transaction costs and "hedge slippage," and this leads to a modified BlackScholes equation; see, for example, [3], [4], [5], [7], [19], [25], [23], [45]. Another method, proposed by Hodges and Neuberger [24], is to price an option so that a utility maximizer is indifferent between either having a certain initial capital for investment or else holding the option but having initial capital reduced by the price of the option. This produces both a price and a hedge, the latter being the difference in the optimal trading strategies in the problem without the option and the problem with the option. This utility-based option pricing is examined in [10], [11], [16]. A formal asymptotic analysis of such an approach appears in [49]. Once again, the methodology developed in this paper suggests how to make this analysis rigorous. We note that in some of these papers the utility function is $U(c)=c^{1-p} /(1-p)$ with $p$ restricted to be in $(0,1)$. We include $p>1$ in our analysis because $p \in(0,1)$ leads to intolerably risky behavior. See Samuelson [42] for the argument in words of one syllable that this is the case even for logarithmic utility $(p=1)$.

The transaction cost problem with multiple stocks was studied by [1], [29]. For a jump diffusion model, see [21]. Transaction cost problems have dual formulations which can shed light on their solutions; see [12], [13], [17] [38]. Other papers which study super-martingales and conditions for no-arbitrage in these models are [12], [26], [27], [28], [35], [48].

In Section 2 we set out the model. Section 3 provides a heuristic expansion of the value function in powers of $\lambda^{1 / 3}$. The key results of Section 3 are proved in Section 4, using viscosity sub- and supersolutions to the HJB equation.

\section{Model set-up and known results}

The set-up of the model is similar to Shreve \& Soner [43]. An agent is given an initial position of $x$ dollars in the money market and $y$ dollars in stock. The stock price is given by $d S_{t}=\alpha S_{t} \mathrm{~d} t+\sigma S_{t} \mathrm{~d} W_{t}$, where $\alpha$ and $\sigma$ are positive constants and $\left\{W_{t}, t \geq 0\right\}$ is a standard Brownian motion on a filtered probability space $\left(\Omega, \mathcal{F},\left\{\mathcal{F}_{t}\right\}_{t \geq 0}, \mathbb{P}\right)$. We assume a constant interest rate $r<\alpha$. The agent must choose a policy consisting of three adapted processes $C, L$, and $M$. The consumption process $C$ is nonnegative and integrable on each finite interval. The processes $L$ and $M$ are nondecreasing and right-continuous with left limits, and $L_{0-}=M_{0-}=0 . L_{t}$ represents the cumulative dollar value of stock purchased up to time $t$, while $M_{t}$ is the cumulative dollar value of stock sold.

Let $X_{t}$ denote the wealth invested in the money market and $Y_{t}$ the wealth 
invested in stock, with $X_{0_{-}}=x, Y_{0_{-}}=y$. The agent's position evolves as

$$
\begin{aligned}
\mathrm{d} X_{t} & =\left(r X_{t}-C_{t}\right) \mathrm{d} t-(1+\lambda) \mathrm{d} L_{t}+(1-\lambda) \mathrm{d} M_{t}, \\
\mathrm{~d} Y_{t} & =\alpha Y_{t} \mathrm{~d} t+\sigma Y_{t} \mathrm{~d} W_{t}+\mathrm{d} L_{t}-\mathrm{d} M_{t} .
\end{aligned}
$$

The constant $\lambda \in(0,1)$ appearing in these equations accounts for proportional transaction costs, which are paid from the money market account.

Define the solvency region

$$
\mathcal{S} \triangleq\{(x, y) ; x+(1+\lambda) y>0, x+(1-\lambda) y>0\} .
$$

The policy $(C, L, M)$ is admissible for $(x, y)$ if $\left(X_{t}, Y_{t}\right)$ given by $(2.1),(2.2)$ is in $\overline{\mathcal{S}}$ for all $t \geq 0$. We denote by $\mathcal{A}(x, y)$ the set of all such policies. We note that $\mathcal{A}(x, y) \neq \emptyset$ if and only if $(x, y) \in \overline{\mathcal{S}}$; see [43], Remarks 2.1 and 2.2 .

We introduce the agent's utility function $U_{p}$ defined for all $c \geq 0$ by $U_{p}(c) \triangleq$ $c^{1-p} /(1-p)$. (An analysis along the lines of this paper is also possible for $U_{0}(c)=\log c$, but we omit that in the interest of brevity.) Let $\beta>0$ be a positive discount rate and define the value function

$$
v(x, y)=\sup _{(C, L, M) \in \mathcal{A}(x, y)} \mathbb{E} \int_{0}^{\infty} e^{-\beta t} U_{p}\left(C_{t}\right) \mathrm{d} t, \quad(x, y) \in \overline{\mathcal{S}} .
$$

This problem when $\lambda=0$ was solved by Merton [40], who determined that the optimal policy always keeps a wealth proportion

$$
\theta_{p}=\frac{1}{p} \frac{\alpha-r}{\sigma^{2}}
$$

in the stock. We call $\theta_{p}$ the Merton proportion. For $\lambda=0$,

$$
v(x, y)=\frac{1}{1-p} A^{-p}(p)(x+y)^{1-p},
$$

where

$$
A(p) \triangleq \frac{\beta-r(1-p)}{p}-\frac{1}{2} \sigma^{2} \theta_{p}^{2}(1-p) .
$$

The optimal consumption in feedback form is $C_{t}=A(p)\left(X_{t}+Y_{t}\right)$.

We assume throughout that $A(p)>0$, which guarantees that the value function for the problem with zero transaction cost is finite.

We introduce the convex dual function $\widetilde{U}_{p}:(0, \infty) \mapsto \mathbb{R}$ defined by

$$
\widetilde{U}_{p}(\tilde{c}) \triangleq \sup _{c>0}\left\{U_{p}(c)-c \tilde{c}\right\}=\frac{p}{1-p} \tilde{c}^{-(1-p) / p} .
$$

The supremum in (2.3) is attained by $c=\tilde{c}^{-1 / p}$.

Shreve and Soner [43] show that the value function is a smooth solution of the Hamilton-Jacobi-Bellman (HJB) equation

$$
\min \left\{\mathcal{L} v-\widetilde{U}_{p}\left(v_{x}\right),-(1-\lambda) v_{x}+v_{y},(1+\lambda) v_{x}-v_{y}\right\}=0,
$$


where the second-order differential operator $\mathcal{L}$ is given by

$$
(\mathcal{L} v)(x, y)=\beta v(x, y)-\frac{1}{2} \sigma^{2} y^{2} v_{y y}(x, y)-\alpha y v_{y}(x, y)-r x v_{x}(x, y) .
$$

The optimal policy can be described in terms of two numbers $0<z_{1}<z_{2}<1 / \lambda$ which define the no-trade region (see [43], Theorem 11.2)

$$
N T \triangleq\left\{(x, y) \in \mathcal{S} ; z_{1}<\frac{y}{x+y}<z_{2}\right\} .
$$

If $Y_{t} /\left(X_{t}+Y_{t}\right)<z_{1}$ one should buy stock in order to bring this ratio to the boundary $y /(x+y)=z_{1}$ of $N T$. If $Y_{t} /\left(X_{t}+Y_{t}\right)>z_{2}$ one should sell stock in order to bring this ratio to the other boundary $y /(x+y)=z_{2}$ of $N T$. For $\theta_{p}<1$ we must have $0<z_{1}<\theta_{p}<z_{2}<1$, so that $N T$ is in the first quadrant. For $\theta_{p}=1$, we have $0<z_{1}<z_{2}=1$. In this paper we show that for $\theta_{p}>1$ and sufficiently small $\lambda, 1<z_{1}<\theta_{p}<z_{2}$, so $N T$ is in the second quadrant.

Power utility functions lead to homotheticity of the value function: for $\gamma>0$,

$$
v(\gamma x, \gamma y)=\gamma^{1-p} v(x, y), \quad(x, y) \in \mathcal{S} .
$$

This is because $(C, L, M) \in \mathcal{A}(x, y) \Leftrightarrow(\gamma C, \gamma L, \gamma M) \in \mathcal{A}(\gamma x, \gamma y)$. Consequently, the problem reduces to that of a single variable. With $\mathcal{T} \triangleq(-1 / \lambda, 1 / \lambda)$, we define

$$
u(z)=v(1-z, z), \quad z \in \mathcal{T} .
$$

In other words, we make the change of variables $z=y /(x+y), 1-z=x /(x+y)$, which maps the solvency region $\mathcal{S}$ onto the interval $\mathcal{T}$. Then

$$
v(x, y)=(x+y)^{1-p} u\left(\frac{y}{x+y}\right), \quad(x, y) \in \mathcal{S} .
$$

The HJB equation corresponding to (2.4) for the function $u(z)$ is

$$
\begin{array}{r}
\min \left\{\mathcal{D} u(z)-\widetilde{U}\left((1-p) u(z)-z u^{\prime}(z)\right), \lambda(1-p) u(z)+(1-\lambda z) u^{\prime}(z),\right. \\
\left.\lambda(1-p) u(z)-(1+\lambda z) u^{\prime}(z)\right\}=0,
\end{array}
$$

where (see [43] p. 681 , substituting $\lambda$ for $\mu, 1+\lambda$ for $1 /(1-\lambda)$ and $1-p$ for $p$ )

$$
\begin{aligned}
\mathcal{D} u(z)= & \left(p A(p)+\frac{1}{2} \sigma^{2} p(1-p)\left(z-\theta_{p}\right)^{2}\right) u(z) \\
& +p \sigma^{2} z(1-z)\left(z-\theta_{p}\right) u^{\prime}(z)-\frac{1}{2} \sigma^{2} z^{2}(1-z)^{2} u^{\prime \prime}(z) .
\end{aligned}
$$

Because $v(x, y)$ is continuous on $\overline{\mathcal{S}}$ and of class $C^{2}$ in $\mathcal{S} \backslash\{(x, y) ; y=0\}$ ([43], Corollary 10.2 and Theorem 11.6), the function $u$ is continuous on $\overline{\mathcal{T}}$, twice 
continuously differentiable on $\mathcal{T}$ except possibly at $z=1$, and satisfies the HJB equation (2.8). Moreover, [43] shows that

$$
\begin{aligned}
\lambda(1-p) u(z)-(1+\lambda z) u^{\prime}(z) & =0, & -\frac{1}{\lambda}<z \leq z_{1}, \\
\mathcal{D} u(z)-\widetilde{U}\left((1-p) u(z)-z u^{\prime}(z)\right) & =0, & z_{1} \leq z \leq z_{2}, \\
\lambda(1-p) u(z)+(1-\lambda z) u^{\prime}(z) & =0, & z_{2} \leq z<\frac{1}{\lambda} .
\end{aligned}
$$

Since the function $u$ is twice continuously differentiable except possibly at $z=1$, at each of $z_{1} \neq 1$ and $z_{2} \neq 1$ two of the above three equations hold. Moreover, $v_{y y}$ is continuous at $x=0$, and $v(0, y)$ satisfies the HJB equation (2.4) ([43], Corollary 10.3). It is also the case that $(1-z)^{2} u^{\prime \prime}(z)$ is continuous for $z \in \mathcal{T}$, and $\lim _{z \rightarrow 1}(z-1)^{2} u^{\prime \prime}(z)=0$ ([43], (A.5)). Thus, two of the above three equations hold at each $z_{i}$ if $z_{i}=1$.

Equations (2.9) and (2.11) are consequences of the directional derivative of $v(x, y)$ being zero in the directions of transaction in the regions in which it is optimal to buy stock and to sell stock, respectively. These equations imply

$$
\begin{aligned}
& u(z)=u\left(z_{1}\right)\left(\frac{1+\lambda z}{1+\lambda z_{1}}\right)^{1-p}, \quad-\frac{1}{\lambda}<z \leq z_{1}, \\
& u(z)=u\left(z_{2}\right)\left(\frac{1-\lambda z}{1-\lambda z_{2}}\right)^{1-p}, \quad z_{2} \leq z<\frac{1}{\lambda} .
\end{aligned}
$$

Lemma 2.1. Assume $p>0, p \neq 1$. For $a>0$ and $b<a$ we have

$$
\begin{aligned}
\widetilde{U}(a-b) & =\frac{p}{1-p}(a-b)\left(a^{-\frac{1}{p}}+\frac{b}{p} a^{-\frac{1+p}{p}}+O\left(b^{2}\right)\right) \\
& =\frac{p}{1-p} a^{-\frac{1-p}{p}}+b a^{-\frac{1}{p}}+O\left(b^{2}\right) .
\end{aligned}
$$

Proof. We write

$$
\widetilde{U}(a-b)=\frac{p}{1-p}(a-b)^{-\frac{1-p}{p}}=\frac{p}{1-p}(a-b)(a-b)^{-\frac{1}{p}} .
$$

A Taylor series expansion yields $(a-b)^{-1 / p}=a^{-1 / p}+\frac{1}{p} b a^{-(1+p) / p}+O\left(b^{2}\right)$, and we get the desired result.

\section{Heuristic derivation by Taylor series}

In this section we derive several terms of a power series expansion of the value function by a heuristic method. One can get an idea on the size of the NT wedge by the following argument. When transaction costs are introduced, it is too expensive for an an agent to keep the proportion of capital in stock equal to $\theta_{p}$. Suppose the agent decides to instead keep the proportion inside an interval centered at $\theta_{p}$ having width $w$. She then incurs an associated cost of transaction 
which is the product $\lambda \ell$ of the transaction cost $\lambda$ and the amount of transacting (local time) $\ell$ accumulated by the state process at the boundaries of the $N T$ wedge. Suppose now that the no-transaction interval has width $\rho w$ for some $\rho>0$. One could multiply the stock volatility by $\rho$, which is equivalent to scaling the Brownian motion by $\rho$, and then the local time on the boundary of the $N T$ wedge would also scale by $\rho$. If one subsequently scales time by $1 / \rho^{2}$, local time is also scaled by $1 / \rho^{2}$, and we have returned to the original volatility. The net effect of these two scalings is to scale local time by $1 / \rho$. In other words, the amount of transacting is inversely proportional to the width of the NT wedge.

On the other hand, by permitting the state process to lie in a wedge rather than at the optimal proportion $\theta_{p}$, the agent loses utility due to displacement from the optimal proportion. This is proportional to the square of the displacement. To see that, one can consider the problem with zero transaction cost and wealth process $V_{t}=X_{t}+Y_{t}$ given by

$$
\mathrm{d} V_{t}=r V_{t} \mathrm{~d} t+(\alpha-r) \theta V_{t} \mathrm{~d} t-c V_{t} \mathrm{~d} t+\sigma \theta V_{t} \mathrm{~d} W(t),
$$

where $\theta$ is a constant proportion of wealth maintained in the stock at all times and $c$ is a constant fraction of wealth being consumed at all times. It is convenient to take $\theta$ to be of the form $\theta_{p}+\epsilon$ and $c$ to be of the form $(1+\delta) A(p) V_{t}$, so that $\epsilon=0$ and $\delta=0$ provide the optimal solution to the zero transaction cost problem. One can then compute

$$
\mathbb{E} V_{t}^{1-p}=V_{0}^{1-p} \exp \{(\beta-A(p)-B(\epsilon)-(1-p) A(p) \delta) t\},
$$

where $B(\epsilon)=\frac{1}{2} p(1-p) \sigma^{2} \theta_{p}^{2} \epsilon^{2}$. This yields expected discounted utility of consumption

$$
\begin{aligned}
& \frac{1}{1-p} \mathbb{E} \int_{0}^{\infty} e^{-\beta t}\left((1+\delta) A(p) V_{t}\right)^{1-p} \mathrm{~d} t \\
& \quad=\frac{1}{1-p} V_{0}^{1-p} A^{-p}(p)(1+\delta)^{1-p}\left(1+\frac{B(\epsilon)}{A(p)}+(1-p) \delta\right)^{-1} .
\end{aligned}
$$

For fixed $\epsilon$, this is maximized by taking $\delta=B(\epsilon) /(p A(p))$, and that value of $\delta$ results in expected utility

$$
\begin{aligned}
& \frac{1}{1-p} \mathbb{E} \int_{0}^{\infty} e^{-\beta t}\left((1+\delta) A(p) V_{t}\right)^{1-p} \mathrm{~d} t \\
& =\frac{1}{1-p} V_{0}^{1-p} A^{-p}(p)\left(1+\frac{1}{2}(1-p) \sigma^{2} \theta_{p}^{2} A^{-1}(p) \epsilon^{2}\right)^{-p} \\
& =\frac{1}{1-p} V_{0}^{1-p} A^{-p}(p)-\frac{p}{2} V_{0}^{1-p} \sigma^{2} \theta_{p}^{2} A^{-1-p}(p) \epsilon^{2}+O\left(\epsilon^{4}\right) .
\end{aligned}
$$

The first term in the last expression is the value function when there is zero transaction costs. The second order $\epsilon^{2}$ term is the loss due to displacement. 
Suppose an agent faced with transaction costs chooses a no-transaction wedge whose width is order $\lambda^{q}$ for some $q>0$. The amount of transacting will be of order $\lambda^{-q}$ and the marginal loss due to transacting will be of order $\lambda^{1-q}$. On the other hand, the marginal loss due to displacement will be of order $\lambda^{2 q}$. The agent chooses $q$ to balance these marginal losses, i.e., chooses $q=\frac{1}{3}$ so that the width of the $N T$ wedge is $\lambda^{1 / 3}$ and the loss in the value function due to the presence of transaction cost $\lambda$ is of order $\lambda^{2 / 3}$.

There is no explicit solution to (2.10) in the interval $\left[z_{1}, z_{2}\right]$. Guided by the above discussion, we thus assume that in this region $u(z)$ has an expansion in powers of $\lambda^{1 / 3}$, and we expect the coefficient of $\lambda^{1 / 3}$ to be zero. In order to work with this expansion, we need to also include the variable $z$, and we do that using powers of $\left(z-\theta_{p}\right)^{1 / 3}$ :

$$
\begin{aligned}
u(z)= & \gamma_{0}-\gamma_{1} \lambda^{\frac{1}{3}}-\gamma_{2} \lambda^{\frac{2}{3}}-\gamma_{3} \lambda-\gamma_{40} \lambda^{\frac{4}{3}}-\gamma_{41}\left(z-\theta_{p}\right) \lambda \\
& -\gamma_{42}\left(z-\theta_{p}\right)^{2} \lambda^{\frac{2}{3}}-\gamma_{43}\left(z-\theta_{p}\right)^{3} \lambda^{\frac{1}{3}}-\gamma_{44}\left(z-\theta_{p}\right)^{4}+O\left(\lambda^{\frac{5}{3}}\right)
\end{aligned}
$$

We argued above that $z-\theta_{p}=O\left(\lambda^{1 / 3}\right)$ for $z \in N T$. This assumption together with (3.1) leads to the expansions

$$
\begin{gathered}
u^{\prime}(z)=-\gamma_{41} \lambda-2 \gamma_{42}\left(z-\theta_{p}\right) \lambda^{\frac{2}{3}}-3 \gamma_{43}\left(z-\theta_{p}\right)^{2} \lambda^{\frac{1}{3}} \\
\quad-4 \gamma_{44}\left(z-\theta_{p}\right)^{3}+O\left(\lambda^{\frac{4}{3}}\right) \\
u^{\prime \prime}(z)=-2 \gamma_{42} \lambda^{\frac{2}{3}}-6 \gamma_{43}\left(z-\theta_{p}\right) \lambda^{\frac{1}{3}}-12 \gamma_{44}\left(z-\theta_{p}\right)^{2}+O(\lambda) .
\end{gathered}
$$

For $z_{1} \leq z \leq z_{2}$ we have

$$
\begin{aligned}
\mathcal{D} u(z)= & p A(p) \gamma_{0}-p A(p) \gamma_{1} \lambda^{\frac{1}{3}}-p A(p) \gamma_{2} \lambda^{\frac{2}{3}}+\frac{1}{2} \sigma^{2} p(1-p)\left(z-\theta_{p}\right)^{2} \gamma_{0} \quad(3.4) \\
& +\sigma^{2} z^{2}(1-z)^{2}\left(\gamma_{42} \lambda^{\frac{2}{3}}+3 \gamma_{43}\left(z-\theta_{p}\right) \lambda^{\frac{1}{3}}+6 \gamma_{44}\left(z-\theta_{p}\right)^{2}\right)+O(\lambda) .
\end{aligned}
$$

Furthermore,

$$
(1-p) u(z)-z u^{\prime}(z)=(1-p) \gamma_{0}-(1-p) \gamma_{1} \lambda^{\frac{1}{3}}-(1-p) \gamma_{2} \lambda^{\frac{2}{3}}+O(\lambda) .
$$

Setting $a=(1-p) \gamma_{0}$ and $b=(1-p) \gamma_{1} \lambda^{1 / 3}+O\left(\lambda^{2 / 3}\right)$ in Lemma 2.1, we obtain

$\widetilde{U}\left((1-p) u(z)-z u^{\prime}(z)\right)=\frac{p}{1-p}\left((1-p) \gamma_{0}\right)^{-\frac{1-p}{p}}+(1-p) \gamma_{1}\left((1-p) \gamma_{0}\right)^{-\frac{1}{p}} \lambda^{\frac{1}{3}}+O\left(\lambda^{\frac{2}{3}}\right)$

Equating first the $O(1)$ terms and then the $O\left(\lambda^{1 / 3}\right)$ terms in (3.4) and (3.6) (see $(2.10))$, we conclude that

$$
\gamma_{0}=\frac{1}{1-p} A^{-p}(p), \quad \gamma_{1}=0
$$

Observe that $\gamma_{0}$ is the value $v(1-z, z)$ for zero transaction costs. 
Since $\gamma_{1}$ is zero, we can now set $b=(1-p) \gamma_{2} \lambda^{2 / 3}+O(\lambda)$ in Lemma 2.1, and obtain (after substituting for $\gamma_{0}$ )

$$
\widetilde{U}\left((1-p) u(z)-z u^{\prime}(z)\right)=\frac{p}{1-p} A^{1-p}(p)+(1-p) \gamma_{2} A(p) \lambda^{\frac{2}{3}}+O(\lambda) .
$$

From (2.12) and (2.13) we observe that $u^{\prime \prime}\left(z_{1}\right)=O\left(\lambda^{2}\right)$ and $u^{\prime \prime}\left(z_{2}\right)=O\left(\lambda^{2}\right)$. From the continuity of $(1-z)^{2} u^{\prime \prime}(z)$ we get from (3.4)

$\mathcal{D} u\left(z_{i}\right)=\frac{p}{1-p} A^{1-p}(p)-p A(p) \gamma_{2} \lambda^{\frac{2}{3}}+\frac{1}{2} \sigma^{2} p\left(z_{i}-\theta_{p}\right)^{2} A^{-p}(p)+O(\lambda), \quad i=1,2$,

where we have omitted the terms in (3.4) arising from $u^{\prime \prime}\left(z_{i}\right)=O\left(\lambda^{2}\right)$. Setting this equal to $(3.7)$ at $z_{i}$ results in $\left(z_{i}-\theta_{p}\right)^{2}=\frac{1}{4} \nu^{2} \lambda^{\frac{2}{3}}+O(\lambda)$, with

$$
\nu=\sqrt{\frac{8}{p \sigma^{2}} A^{1+p}(p) \gamma_{2}} .
$$

We thus have

$$
z_{1}-\theta_{p}=-\frac{1}{2} \nu \lambda^{\frac{1}{3}}+O\left(\lambda^{\frac{2}{3}}\right), \quad z_{2}-\theta_{p}=\frac{1}{2} \nu \lambda^{\frac{1}{3}}+O\left(\lambda^{\frac{2}{3}}\right) .
$$

We may also equate the $\lambda^{2 / 3}$ terms in (3.4) and (3.7) at $z=\theta_{p}, z=z_{1}$, and $z=z_{2}$, and substitute for $z_{i}-\theta_{p}$ from (3.9), to obtain

$$
\begin{aligned}
& A(p) \gamma_{2}=\sigma^{2} \theta_{p}^{2}\left(1-\theta_{p}\right)^{2} \gamma_{42}, \\
& A(p) \gamma_{2}=\frac{1}{8} \sigma^{2} p A^{-p}(p) \nu^{2}+\sigma^{2} \theta_{p}^{2}\left(1-\theta_{p}\right)^{2}\left(\gamma_{42}-\frac{3}{2} \gamma_{43} \nu+\frac{3}{2} \gamma_{44} \nu^{2}\right), \\
& A(p) \gamma_{2}=\frac{1}{8} \sigma^{2} p A^{-p}(p) \nu^{2}+\sigma^{2} \theta_{p}^{2}\left(1-\theta_{p}\right)^{2}\left(\gamma_{42}+\frac{3}{2} \gamma_{43} \nu+\frac{3}{2} \gamma_{44} \nu^{2}\right),
\end{aligned}
$$

which implies

$$
\gamma_{42}=\frac{A(p) \gamma_{2}}{\sigma^{2} \theta_{p}^{2}\left(1-\theta_{p}\right)^{2}}, \quad \gamma_{43}=0, \quad \gamma_{44}=-\frac{p A^{-p}(p)}{12 \theta_{p}^{2}\left(1-\theta_{p}\right)^{2}} .
$$

Finally, we observe from (2.12) and from (3.1) that

$$
u^{\prime}\left(z_{1}\right)=\frac{\lambda(1-p)}{1+\lambda z_{1}} u\left(z_{1}\right)=\lambda(1-p) u\left(z_{1}\right)+O\left(\lambda^{2}\right)=A^{-p}(p) \lambda+O\left(\lambda^{\frac{5}{3}}\right),
$$

and similarly for $u^{\prime}\left(z_{2}\right)$. On the other hand, (3.2) and (3.9) imply that

$$
\begin{aligned}
& u^{\prime}\left(z_{1}\right)=-\gamma_{41} \lambda+\gamma_{42} \nu \lambda-\frac{3}{4} \gamma_{43} \nu^{2} \lambda+\frac{1}{2} \gamma_{44} \nu^{3} \lambda+O\left(\lambda^{\frac{4}{3}}\right), \\
& u^{\prime}\left(z_{2}\right)=\gamma_{41} \lambda+\gamma_{42} \nu \lambda+\frac{3}{4} \gamma_{43} \nu^{2} \lambda+\frac{1}{2} \gamma_{44} \nu^{3} \lambda+O\left(\lambda^{\frac{4}{3}}\right) .
\end{aligned}
$$


It follows that

$$
-\gamma_{41}+\gamma_{42} \nu-\frac{3}{4} \gamma_{43} \nu^{2}+\frac{1}{2} \gamma_{44} \nu^{3}=A^{-p}(p)=\gamma_{41}+\gamma_{42} \nu+\frac{3}{4} \gamma_{43} \nu^{2}+\frac{1}{2} \gamma_{44} \nu^{3} .
$$

Since $\gamma_{43}=0$ (see (3.10)), we conclude

$$
\gamma_{42} \nu+\frac{1}{2} \gamma_{44} \nu^{3}=A^{-p}(p), \quad \gamma_{41}=0
$$

We have thus written the value function as

$$
u(z)=\frac{1}{1-p} A^{-p}(p)-\gamma_{2} \lambda^{\frac{2}{3}}+O(\lambda) .
$$

Furthermore, we can solve for $\gamma_{2}$ and the width of the $N T$ interval $\nu$ from equations (3.8), (3.10), and (3.11) to obtain

$$
\gamma_{2}=\left(\frac{9}{32} p \theta_{p}^{4}\left(1-\theta_{p}\right)^{4}\right)^{\frac{1}{3}} A^{-1-p}(p) \sigma^{2}, \quad \nu=\left(\frac{12}{p} \theta_{p}^{2}\left(1-\theta_{p}\right)^{2}\right)^{\frac{1}{3}} .
$$

\section{Rigorous asymptotic expansion}

Definition 4.1. Let $w: \mathcal{T} \rightarrow \mathbb{R}$ be continuous, taking values in $(0, \infty)$ if $0<$ $p<1$ and taking values in $(-\infty, 0)$ if $p>1$. Assume there are two points $0<\zeta_{1}<\zeta_{2}<1 / \lambda$ such that

$$
\begin{aligned}
& w(z)=w\left(\zeta_{1}\right)\left(\frac{1+\lambda z}{1+\lambda \zeta_{1}}\right)^{1-p}, \quad-\frac{1}{\lambda}<z \leq \zeta_{1}, \\
& w(z)=w\left(\zeta_{2}\right)\left(\frac{1-\lambda z}{1-\lambda \zeta_{2}}\right)^{1-p}, \quad \zeta_{2} \leq z<\frac{1}{\lambda} .
\end{aligned}
$$

Assume further that $w$ is of class $C^{2}$ on $\left(\zeta_{1}, \zeta_{2}\right)$ and the one-sided limits of $w^{\prime \prime}$ exist at the endpoints of this interval. If

$$
\begin{aligned}
\lambda(1-p) w(z)+(1-\lambda z) w^{\prime}(z) & \geq 0, \quad-\frac{1}{\lambda}<z<\zeta_{2}, \\
\mathcal{D} w(z)-\widetilde{U}\left((1-p) w(z)-z w^{\prime}(z)\right) & \geq 0, \quad-\frac{1}{\lambda}<z<\frac{1}{\lambda}, \\
\lambda(1-p) w(z)-(1+\lambda z) w^{\prime}(z) & \geq 0, \quad \zeta_{1}<z<\frac{1}{\lambda},
\end{aligned}
$$

we say $\left(w, \zeta_{1}, \zeta_{2}\right)$ is a supersolution triple. If (4.1), (4.2) and the reverse of inequality (4.4) for $\zeta_{1}<z<\zeta_{2}$ hold, we say $\left(w, \zeta_{1}, \zeta_{2}\right)$ is a subsolution triple.

If $\left(w, \zeta_{1}, \zeta_{2}\right)$ is a supersolution (subsolution) triple, then

$$
\varphi(x, y) \triangleq(x+y)^{1-p} w(y /(x+y)), \quad(x, y) \in \mathcal{S}
$$

is a supersolution (subsolution) of (2.4). 
Lemma 4.2. Assume $p>0, p \neq 1$. If $\left(w, \zeta_{1}, \zeta_{2}\right)$ is a supersolution (subsolution) triple, then $w(z) \geq u(z)(w(z) \leq u(z))$ for $z \in \mathcal{T}$.

Proof. For $0<p<1$, this follows from [43], Comparison Theorem A.3. We give the proof for $p>1$.

Let $\left(w, \zeta_{1}, \zeta_{2}\right)$ be a supersolution triple, and let $\varphi$ be given by (4.6). In light of (2.7), is suffices to prove that $\varphi\left(x_{0}, y_{0}\right) \geq v\left(x_{0}, y_{0}\right)$ for fixed but arbitrary $\left(x_{0}, y_{0}\right) \in \mathcal{S}$. Following the construction of Theorem 9.2 of [43], we let $(C, L, M)$ be an optimal policy for this initial condition, i.e., with $\left(X_{t}, Y_{t}\right)$ given by $(2.1)$, $(2.2)$, we have $(X(0), Y(0)) \in \partial N T$ if $\left(x_{0}, y_{0}\right) \notin N T,\left(X_{t}, Y_{t}\right) \in \overline{N T}$ for all $t \geq 0$, and

$$
\begin{gathered}
L_{t}=\int_{0}^{t} \mathbb{I}_{\left\{Y_{s} /\left(X_{s}+Y_{s}\right)=z_{1}\right\}} \mathrm{d} L_{s}, \quad M_{t}=\int_{0}^{t} \mathbb{I}_{\left\{Y_{s} /\left(X_{s}+Y_{s}\right)=z_{2}\right\}} \mathrm{d} M_{s}, \\
C_{t}=\left(v_{x}\left(X_{t}, Y_{t}\right)\right)^{-1 / p}, \quad t \geq 0 .
\end{gathered}
$$

Then $v\left(x_{0}, y_{0}\right)=\mathbb{E} \int_{0}^{\infty} e^{-\beta t} U_{p}\left(C_{t}\right) \mathrm{d} t$.

The function $\varphi$ is of class $C^{2}$ in $\mathcal{S}$ except possibly on the lines $y /(x+y)=\zeta_{i}$, $i=1,2$. We can mollify $\varphi$ to obtain a $C^{2}$ function, apply Itô's rule to this $C^{2}$ function, and then pass to the limit. On the two lines where $\varphi$ may not be $C^{2}$, $\left(X_{t}, Y_{t}\right)$ spends Lebesgue-measure zero time and we will obtain Itô's rule for $\varphi\left(X_{t}, Y_{t}\right)$. An exception would be if $\zeta_{2}=z_{2}=1$, because in this case $\left(X_{t}, Y_{t}\right)$ remains on the $y$-axis once the $y$-axis is reached. A direct computation shows, however, that $\varphi_{y y}(x, y)$ is continuous there, even if $\zeta_{2}=z_{2}=1$, and hence we will still obtain Itô's rule. Therefore,

$$
\begin{aligned}
& \left(e^{-\beta t} \varphi\left(X_{t}, Y_{t}\right)\right) \\
& =-e^{-\beta t}\left[\mathcal{L} \varphi\left(X_{t}, Y_{t}\right) \mathrm{d} t+C_{t} \varphi_{x}\left(X_{t}, Y_{t}\right)+\left((1+\lambda) \varphi_{x}\left(X_{t}, Y_{t}\right)-\varphi_{y}\left(X_{t}, Y_{t}\right)\right) \mathrm{d} L_{t}\right. \\
& \left.\quad+\left(-(1-\lambda) \varphi_{x}\left(X_{t}, Y_{t}\right)+\varphi_{y}\left(X_{t}, Y_{t}\right)\right) \mathrm{d} M_{t}\right]+e^{-\beta t} \varphi_{y}\left(X_{t}, Y_{t}\right) \sigma \mathrm{d} W_{t} \\
& \leq-e^{-\beta t} U_{p}\left(C_{t}\right) \mathrm{d} t+e^{-\beta t} \varphi_{y}\left(X_{t}, Y_{t}\right) \sigma \mathrm{d} W_{t},
\end{aligned}
$$

where we have used the supersolution property, the fact that $\mathcal{L} \varphi=\mathcal{D} u$, and the fact (see(2.3)) that

$$
\widetilde{U}_{p}\left(\varphi_{x}\left(X_{t}, Y_{t}\right)\right) \geq U_{p}\left(C_{t}\right)-C_{t} \varphi_{x}\left(X_{t}, Y_{t}\right) .
$$

We want to integrate (4.7) and eventually argue that the expected value of the Itô integral is zero. Define $\tau_{n}=\inf \left\{t \geq 0 ;\left|X_{t}+Y_{t}\right| \leq 1 / n\right\}$, to obtain

$$
\begin{aligned}
\int_{0}^{n \wedge \tau_{n}} e^{-\beta t} U_{p}\left(C_{t}\right) \mathrm{d} t+e^{-\beta\left(n \wedge \tau_{n}\right)} \varphi\left(X_{n \wedge \tau_{n}}, Y_{n \wedge \tau_{n}}\right) \\
\leq \varphi\left(x_{0}, y_{0}\right)+\sigma \int_{0}^{n \wedge \tau_{n}} e^{-\beta t} \varphi_{y}\left(X_{t}, Y_{t}\right) \mathrm{d} W_{t} .
\end{aligned}
$$

We can perform a similar analysis for the value function. The function $v$ is $C^{2}$ except on the $y$-axis. The $x$-axis is not in $\overline{N T}$, which contains $\left(X_{t}, Y_{t}\right)$ for all 
$t \geq 0$. At the $y$-axis, $v_{y y}$ is continuous ([43], Corollary 10.3). This permits us to compute the differential of $v\left(X_{t}, Y_{t}\right)$, and similarly to (4.9) we obtain

$$
\begin{aligned}
\int_{0}^{n \wedge \tau_{n}} e^{-\beta t} U_{p}\left(C_{t}\right) \mathrm{d} t & +e^{-\beta\left(n \wedge \tau_{n}\right)} v\left(X_{n \wedge \tau_{n}}, Y_{n \wedge \tau_{n}}\right) \\
= & v\left(x_{0}, y_{0}\right)+\sigma \int_{0}^{n \wedge \tau_{n}} e^{-\beta t} v_{y}\left(X_{t}, Y_{t}\right) \mathrm{d} W_{t} .
\end{aligned}
$$

As $n \rightarrow \infty$, we have either $\tau_{n} \rightarrow \infty$ or $\tau_{n} \rightarrow \tau_{0} \triangleq \inf \left\{t \geq 0: X_{t}=Y_{t}=0\right\}<\infty$. On the set $\left\{\lim _{n \rightarrow \infty} \tau_{n}=\tau_{0}<\infty\right\}$, equation (4.10), the inequality $U_{p} \leq 0$ and the fact that $v(0,0)=-\infty$ imply $\int_{0}^{\tau_{0}} e^{-\beta t} v_{y}\left(X_{t}, Y_{t}\right) \mathrm{d} W_{t}=-\infty$. This is impossible, because Itô integrals are either finite or have $\lim \sup =-\lim \inf =$ $\infty$ (see [32], Chap. 3, Problem 4.11 and p. 232). We conclude that $\tau_{n} \rightarrow \infty$ almost surely. Taking expectations in (4.10) and letting $n \rightarrow \infty$, we obtain

$$
\lim _{n \rightarrow \infty} \mathbb{E} e^{-\beta\left(n \wedge \tau_{n}\right)} v\left(X_{n \wedge \tau_{n}}, Y_{n \wedge \tau_{n}}\right)=v\left(x_{0}, y_{0}\right)-\mathbb{E} \int_{0}^{\infty} e^{-\beta t} U_{p}\left(C_{t}\right) \mathrm{d} t=0 .
$$

Because of (2.7), (4.6) and the boundedness of $u$ and $w$ for $z \in N T$, either $\varphi(x, y)=0$, or there are constants $c_{1}$ and $c_{2}$ such that

$$
c_{1} \varphi(x, y) \leq v(x, y) \leq c_{2} \varphi(x, y) \text { whenever } z_{1} \leq \frac{y}{x+y} \leq z_{2} .
$$

From (4.11) we conclude that $\lim _{n \rightarrow \infty} \mathbb{E} e^{-\beta\left(n \wedge \tau_{n}\right)} \varphi\left(X_{n \wedge \tau_{n}}, Y_{n \wedge \tau_{n}}\right)=0$. Taking expectations and the limit in (4.9), we have $v\left(x_{0}, y_{0}\right)=\mathbb{E} \int_{0}^{\infty} e^{-\beta t} U_{p}\left(C_{t}\right) \mathrm{d} t \leq$ $\varphi\left(x_{0}, y_{0}\right)$, as desired.

Now let $\left(w, \zeta_{1}, \zeta_{2}\right)$ be a subsolution triple. We show for fixed but arbitrary $\left(x_{0}, y_{0}\right) \in \mathcal{S}$ that $\varphi\left(x_{0}, y_{0}\right) \leq v\left(x_{0}, y_{0}\right)$. This time we construct a (suboptimal) policy $(C, L, M)$ for which $Y(0) /(X(0)+Y(0))$ is either $\zeta_{1}$ or $\zeta_{2}$ if $y_{0} /\left(x_{0}+y_{0}\right) \notin$ $\left(\zeta_{1}, \zeta_{2}\right),\left(X_{t}, Y_{t}\right) \in\left[\zeta_{1}, \zeta_{2}\right]$ for all $t \geq 0$, and

$$
\begin{gathered}
L_{t}=\int_{0}^{t} \mathbb{I}_{\left\{Y_{s} /\left(X_{s}+Y_{s}\right)=\zeta_{1}\right\}} d L_{s}, \quad M_{t}=\int_{0}^{t} \mathbb{I}_{\left\{Y_{s} /\left(X_{s}+Y_{s}\right)=\zeta_{2}\right\}} d M_{s}, \\
C_{t}=\left(\varphi_{x}\left(X_{t}, Y_{t}\right)\right)^{-1 / p}, \quad t \geq 0 .
\end{gathered}
$$

For this policy, we have the reverse equality in $(4.9) .^{2}$ Using this inequality in place of (4.10), we argue as before that $\lim _{n \rightarrow \infty} \tau_{n}=\infty$ almost surely. $\varphi \leq 0$, (4.9) with the inequality reversed implies $\mathbb{E} \int_{0}^{n \wedge \tau_{n}} e^{-\beta t} U_{p}\left(C_{t}\right) \mathrm{d} t \geq \varphi\left(x_{0}, y_{0}\right)$ and letting $n \rightarrow \infty$, we obtain $v\left(x_{0}, y_{0}\right) \geq \mathbb{E} \int_{0}^{\infty} e^{-\beta t} U_{p}\left(C_{t}\right) \mathrm{d} t \geq \varphi\left(x_{0}, y_{0}\right)$.

\footnotetext{
${ }^{2}$ We used the optimal policy for the supersolution argument to get equality in (4.10). For the subsolution argument we get the appropriate inequality in (4.10) by using a suboptimal policy. However, we need to pick trading policy such that the terms containing the integrals $\mathrm{d} L_{t}$ and $\mathrm{d} M_{t}$ in (4.7) are zero, and consumption policy so that we get equality in (4.8).
} 
Theorem 4.3. Assume $p>0, p \neq 1$ and $A(p)>0$. Then the value function $u$ satisfies

$$
u\left(\theta_{p}\right)=\frac{1}{1-p} A^{-p}(p)-\left(\frac{9}{32} p \theta_{p}^{4}\left(1-\theta_{p}\right)^{4}\right)^{\frac{1}{3}} A^{-1-p}(p) \sigma^{2} \lambda^{\frac{2}{3}}+O(\lambda) .
$$

Proof. We assume $\theta_{p} \neq 1$. For $\theta_{p}=1$ see Remark 4.6.

Step 1: Choice of constants and variables

We recall the constants $\gamma_{2}$ and $\nu$ of $(3.12)$. Set $\xi=\sqrt{\frac{2}{3}(1-p) \gamma_{2} A^{p}(p)+B}$, where $B$ is a constant chosen to make the expression under the square root positive. We next define

$$
h(\delta)=\frac{3}{2} \delta^{2} \lambda^{\frac{2}{3}}-\frac{1}{\nu^{2}} \cdot \delta^{4}+\frac{3}{2} B \delta^{2} \lambda^{\frac{2}{3}}
$$

and choose a positive constant $M$ satisfying

$$
M>\theta_{p} A^{-p}(p)+\frac{1}{4} \sigma^{2} p \nu^{2} \xi^{2} A^{-p-1}(p) .
$$

We define functions

$$
\begin{gathered}
f_{1}^{ \pm}(\delta)=\nu \lambda^{\frac{1}{3}}-(1-p) \nu \gamma_{2} A^{p}(p) \lambda \pm(1-p) \nu M A^{p}(p) \lambda^{\frac{4}{3}}-(1-p) h(-\delta) \lambda^{\frac{1}{3}} \\
+\left(\lambda^{-\frac{2}{3}}+\left(\theta_{p}-\delta\right) \lambda^{\frac{1}{3}}\right) h^{\prime}(-\delta), \\
f_{2}^{ \pm}(\delta)=\nu \lambda^{\frac{1}{3}}-(1-p) \nu \gamma_{2} A^{p}(p) \lambda \pm(1-p) \nu M A^{p}(p) \lambda^{\frac{4}{3}}-(1-p) h(\delta) \lambda^{\frac{1}{3}} \\
+\left(-\lambda^{-\frac{2}{3}}+\left(\theta_{p}+\delta\right) \lambda^{\frac{1}{3}}\right) h^{\prime}(\delta) .
\end{gathered}
$$

It is shown in Appendix A that there are numbers

$$
\delta_{1}^{ \pm}=\frac{1}{2} \nu \lambda^{\frac{1}{3}}\left(1-\xi \lambda^{\frac{1}{3}}\right)+o\left(\lambda^{\frac{2}{3}}\right), \quad \delta_{2}^{ \pm}=\frac{1}{2} \nu \lambda^{\frac{1}{3}}\left(1-\xi \lambda^{\frac{1}{3}}\right)+o\left(\lambda^{\frac{2}{3}}\right)
$$

satisfying $f_{i}^{ \pm}\left(\delta_{i}^{ \pm}\right)=0, i=1,2$.

Step 2: Construction of super/subsolutions.

Choose $\lambda>0$ small enough that $\zeta_{1}^{ \pm} \triangleq \theta_{p}-\delta_{1}^{ \pm}$and $\zeta_{2}^{ \pm} \triangleq \theta_{p}+\delta_{2}^{ \pm}$all lie in $(0,1 / \lambda)$. (We have $\theta_{p}>0$ since $\alpha>r$.) Define

$$
w_{ \pm}(z)=\left\{\begin{array}{l}
\left(\frac{A^{-p}(p)}{1-p}-\gamma_{2} \lambda^{\frac{2}{3}} \pm M \lambda-\frac{A^{-p}(p)}{\nu} h\left(\zeta_{1}^{ \pm}-\theta_{p}\right)\right)\left(\frac{1+\lambda z}{1+\lambda \zeta_{1}^{ \pm}}\right), \quad-\frac{1}{\lambda} \leq z \leq \zeta_{1}^{ \pm}, \\
\frac{1}{1-p} A^{-p}(p)-\gamma_{2} \lambda^{\frac{2}{3}} \pm M \lambda-\frac{A^{-p}(p)}{\nu} h\left(z-\theta_{p}\right), \quad \zeta_{1}^{ \pm} \leq z \leq \zeta_{2}^{ \pm}, \\
\left(\frac{A^{-p}(p)}{1-p}-\gamma_{2} \lambda^{\frac{2}{3}} \pm M \lambda-\frac{A^{-p}(p)}{\nu} h\left(\zeta_{2}^{ \pm}-\theta_{p}\right)\right)\left(\frac{1-\lambda z}{1-\lambda \zeta_{2}^{ \pm}}\right), \quad \zeta_{2}^{ \pm} \leq z \leq \frac{1}{\lambda} .
\end{array}\right.
$$

The reader can verify that if $M$ were zero, then in the region $\left[\zeta_{1}^{ \pm}, \zeta_{2}^{ \pm}\right]$the formula for $w_{ \pm}(z)$ agrees with the power series expansion

$$
\gamma_{0}-\gamma_{2} \lambda^{\frac{2}{3}}-\gamma_{42}\left(z-\theta_{p}\right)^{2} \lambda^{\frac{2}{3}}-\gamma_{44}\left(z-\theta_{p}\right)^{4},
$$


where the coefficients $\gamma_{0}, \gamma_{2}, \gamma_{42}$, and $\gamma_{44}$ are those worked out in the previous section. The term $\pm M \lambda$ in the definition of $w_{ \pm}$will be used to create supersolution and subsolution triples. We have the derivative formula

$$
w_{ \pm}^{\prime}(z)=\left\{\begin{array}{lr}
\frac{\lambda}{1+\lambda z}(1-p) w_{ \pm}(z), & -\frac{1}{\lambda}<z \leq \zeta_{1}^{ \pm} \\
-\nu^{-1} A^{-p}(p) h^{\prime}\left(z-\theta_{p}\right), & \zeta_{1}^{ \pm} \leq z \leq \zeta_{2}^{ \pm} \\
-\frac{\lambda}{1-\lambda z}(1-p) w_{ \pm}(z), & \zeta_{2}^{ \pm} \leq z<\frac{1}{\lambda}
\end{array}\right.
$$

The equations $f_{1}^{ \pm}\left(\delta_{1}\right)=0$ and $f_{2}^{ \pm}\left(\delta_{2}\right)=0$ guarantee that $w_{ \pm}^{\prime}$ is defined and continuous at $\zeta_{1}^{ \pm}$and $\zeta_{2}^{ \pm}$. We also have

$$
w_{ \pm}^{\prime \prime}(z)=\left\{\begin{array}{lc}
-\frac{\lambda^{2}}{(1+\lambda z)^{2}} p(1-p) w_{ \pm}(z), & -\frac{1}{\lambda}<z<\zeta_{1}^{ \pm}, \\
-\nu^{-1} A^{-p}(p) h^{\prime \prime}\left(z-\theta_{p}\right), & \zeta_{1}^{ \pm}<z<\zeta_{2}^{ \pm}, \\
-\frac{\lambda^{2}}{(1-\lambda z)^{2}} p(1-p) w_{ \pm}(z), & \zeta_{2}^{ \pm}<z<\frac{1}{\lambda} .
\end{array}\right.
$$

The function $w_{ \pm}(z)$ is $\mathcal{C}^{2}$ except at $\zeta_{1}^{ \pm}$and $\zeta_{2}^{ \pm}$, and at these two points, the one-sided second derivatives exist and equal the respective one-sided limits of the second derivatives.

Step 3: Verification that $\left(w_{-}, \zeta_{1}^{-}, \zeta_{2}^{-}\right)$is a subsolution triple.

It suffices to verify

$$
\mathcal{D} w_{-}(z)-\widetilde{U}\left((1-p) w_{-}(z)-z w_{-}^{\prime}(z)\right) \leq 0, \quad \zeta_{1}^{-}<z<\zeta_{2}^{-} .
$$

To do this, we simultaneously work with both $w_{-}$and $w_{+}$. We thereby develop an inequality for $w_{+}$needed in the subsequent supersolution verification. We use the facts that $z-\theta_{p}=O\left(\lambda^{1 / 3}\right)$, so $h\left(z-\theta_{p}\right)=O\left(\lambda^{4 / 3}\right), h^{\prime}\left(z-\theta_{p}\right)=O(\lambda)$.

For $\zeta_{1}^{ \pm}<z<\zeta_{2}^{ \pm}$we have $(1-p) w_{ \pm}(z)-z w_{ \pm}^{\prime}(z)=a-b$, where $a=A^{-p}(p)$ and $b=(1-p) \gamma_{2} \lambda^{2 / 3} \mp(1-p) M \lambda-A^{-p}(p) \nu^{-1} z h^{\prime}\left(z-\theta_{p}\right)+O\left(\lambda^{4 / 3}\right)$. Lemma 2.1 implies

$$
\begin{aligned}
& \widetilde{U}\left((1-p) w_{ \pm}(z)-z w_{ \pm}^{\prime}(z)\right) \\
& =\frac{p}{1-p} A^{1-p}(p)+A(p)\left[(1-p) \gamma_{2} \lambda^{\frac{2}{3}} \mp(1-p) M \lambda-\frac{A^{-p}(p)}{\nu} z h^{\prime}\left(z-\theta_{p}\right)\right] \\
& \quad+O\left(\lambda^{\frac{4}{3}}\right) \\
& \quad p A(p) w(z)+\gamma_{2} A(p) \lambda^{\frac{2}{3}} \mp M \lambda-\frac{A^{1-p}(p)}{\nu} z h^{\prime}\left(z-\theta_{p}\right)+O\left(\lambda^{\frac{4}{3}}\right) .
\end{aligned}
$$

Therefore,

$$
\begin{aligned}
& \mathcal{D} w_{ \pm}(z)-\widetilde{U}\left((1-p) w_{ \pm}(z)-z w_{ \pm}^{\prime}(z)\right) \\
&=\frac{1}{2} \sigma^{2} p(1-p)\left(z-\theta_{p}\right)^{2} w_{ \pm}(z)-\frac{1}{2} \sigma^{2} z^{2}(1-z)^{2} w_{ \pm}^{\prime \prime}(z) \\
& \quad-\gamma_{2} A(p) \lambda^{\frac{2}{3}} \pm M \lambda+\frac{1}{\nu} A^{1-p}(p) z h^{\prime}\left(z-\theta_{p}\right)+O\left(\lambda^{\frac{4}{3}}\right)
\end{aligned}
$$




$$
\begin{gathered}
=\frac{1}{2} \sigma^{2} p A^{-p}(p)\left(z-\theta_{p}\right)^{2}+\frac{1}{2 \nu} \sigma^{2} z^{2}(1-z)^{2} A^{-p}(p) h^{\prime \prime}\left(z-\theta_{p}\right) \\
-\gamma_{2} A(p) \lambda^{\frac{2}{3}} \pm M \lambda+\frac{1}{\nu} A^{1-p}(p) z h^{\prime}\left(z-\theta_{p}\right)+O\left(\lambda^{\frac{4}{3}}\right) .
\end{gathered}
$$

Writing $z=\theta_{p}+\left(z-\theta_{p}\right)$ and $1-z=1-\theta_{p}-\left(z-\theta_{p}\right)$, we derive the relation

$$
z^{2}(1-z)^{2}=\theta_{p}^{2}\left(1-\theta_{p}\right)^{2}+2 \theta_{p}\left(1-\theta_{p}\right)\left(1-2 \theta_{p}\right)\left(z-\theta_{p}\right)+O\left(\lambda^{\frac{2}{3}}\right) .
$$

Using this and the formulas $h^{\prime}(\delta)=3 \delta \lambda^{2 / 3}-4 \delta^{3} / \nu^{2}+3 B \delta \lambda^{2 / 3}, h^{\prime \prime}(\delta)=3 \lambda^{2 / 3}-$ $12 \delta^{2} / \nu^{2}+3 B \lambda^{2 / 3}$, we obtain

$$
\begin{aligned}
\mathcal{D} w_{ \pm}(z)-\widetilde{U} & \left((1-p) w_{ \pm}(z)-z w_{ \pm}^{\prime}(z)\right) \\
=\left[\frac{1}{2} \sigma^{2} p A^{-p}(p)-\frac{6}{\nu^{3}} \sigma^{2} \theta_{p}^{2}\left(1-\theta_{p}\right)^{2} A^{-p}(p)\right]\left(z-\theta_{p}\right)^{2} & \\
+ & {\left[\frac{3}{2 \nu} \sigma^{2} \theta_{p}^{2}\left(1-\theta_{p}\right)^{2} A^{-p}(p)-\gamma_{2} A(p)\right] \lambda^{\frac{2}{3}} } \\
& +\sigma^{2} \theta_{p}\left(1-\theta_{p}\right)\left(1-2 \theta_{p}\right) A^{-p}(p)\left[\frac{3\left(z-\theta_{p}\right) \lambda^{\frac{2}{3}}}{\nu}-\frac{12\left(z-\theta_{p}\right)^{3}}{\nu^{3}}\right] \\
& +\theta_{p} A^{-p}(p)\left[\frac{3\left(z-\theta_{p}\right) \lambda^{\frac{2}{3}}}{\nu}-\frac{4\left(z-\theta_{p}\right)^{3}}{\nu^{3}}\right] \pm M \lambda+O\left(\lambda^{\frac{4}{3}}\right) .
\end{aligned}
$$

The definitions of $\nu$ and $\gamma_{2}$ imply that the first two terms on the right-hand side are zero. Because

$$
\frac{z-\theta_{p}}{\nu}=\frac{1}{2} \lambda^{\frac{1}{3}}+O\left(\lambda^{\frac{2}{3}}\right)
$$

the third term is $O\left(\lambda^{4 / 3}\right)$, and we can simplify the fourth term to obtain

$$
\mathcal{D} w_{ \pm}(z)-\widetilde{U}\left((1-p) w_{ \pm}(z)-z w_{ \pm}^{\prime}(z)\right)=\theta_{p} A^{-p}(p) \lambda \pm M \lambda+O\left(\lambda^{\frac{4}{3}}\right)
$$

for $z_{1}^{ \pm}<z<z_{2}^{ \pm}$. From (4.13) we have (4.17) for all sufficiently small $\lambda>0$. This completes the verification that $\left(w_{-}, \zeta_{1}^{-}, \zeta_{2}^{-}\right)$is a subsolution triple.

Step 4: Verification that $\left(w_{+}, \zeta_{1}^{+}, \zeta_{2}^{+}\right)$is a supersolution triple Step 4a: Interval $\left(-1 / \lambda, \zeta_{1}^{+}\right)$.

We must show that that (4.3) and (4.4) hold in this interval. Since $(1-$ $p) w_{+}(z)>0$ for sufficiently small $\lambda>0$, so is $w_{+}^{\prime}(z)$, and thus (4.3) holds.

It remains to verify that for sufficiently small $\lambda$

$$
\mathcal{D} w_{+}(z)-\widetilde{U}\left((1-p) w_{+}(z)-z w_{+}^{\prime}(z)\right) \geq 0, \quad-\frac{1}{\lambda}<z<\zeta_{1}^{+} .
$$


In this interval,

$$
\begin{aligned}
(1-p) w_{+}(z)-z w_{+}^{\prime}(z)= & \frac{1-p}{1+\lambda z} w_{+}(z) \\
= & \frac{1}{1+\lambda \zeta_{1}^{+}}\left(\frac{1+\lambda z_{1}^{+}}{1+\lambda z}\right)^{p}\left(A^{-p}(p)-(1-p) \gamma_{2} \lambda^{\frac{2}{3}}\right. \\
& \left.\quad+(1-p) M \lambda-\frac{1}{\nu}(1-p) A^{-p}(p) h\left(\zeta_{1}^{+}-\theta_{p}\right)\right) .
\end{aligned}
$$

Using the first equality in Lemma 2.1 and the equality $\widetilde{U}(\alpha \tilde{c})=\alpha^{-(1-p) / p} \widetilde{U}(\tilde{c})$, we get

$$
\begin{aligned}
\widetilde{U}\left((1-p) w_{+}(z)-z w_{+}^{\prime}(z)\right) \\
=\left(1+\lambda z_{1}^{+}\right)^{\frac{1-p}{p}}\left(\frac{1+\lambda z}{1+\lambda \zeta_{1}^{+}}\right)^{1-p} \frac{p}{1-p} \\
\times\left(A^{-p}(p)-(1-p) \gamma_{2} \lambda^{\frac{2}{3}}+(1-p) M \lambda-\frac{1-p}{\nu} A^{-p}(p) h\left(\zeta_{1}^{+}-\theta_{p}\right)\right) \\
\quad \times\left(A(p)+\frac{1-p}{p} \gamma_{2} A^{p+1}(p) \lambda^{\frac{2}{3}}-\frac{1-p}{p} M A^{p+1}(p) \lambda+O\left(\lambda^{\frac{4}{3}}\right)\right) \\
=\left(1+\lambda \zeta_{1}^{+}\right)^{\frac{1-p}{p}} w_{+}(z) \\
\quad \times\left(p A(p)+(1-p) \gamma_{2} A^{p+1}(p) \lambda^{\frac{2}{3}}-(1-p) M A^{p+1}(p) \lambda+O\left(\lambda^{\frac{4}{3}}\right)\right) .
\end{aligned}
$$

$\operatorname{But}\left(1+\lambda \zeta_{1}^{+}\right)^{(1-p) / p}=1+\frac{1-p}{p} \lambda \zeta_{1}^{+}+O\left(\lambda^{2}\right)=1+\frac{1-p}{p} \lambda \theta_{p}+O\left(\lambda^{4 / 3}\right)$, and thus

$$
\begin{gathered}
\widetilde{U}\left((1-p) w_{+}(z)-z w_{+}^{\prime}(z)\right) \\
=w_{+}(z)\left(p A(p)+(1-p) \gamma_{2} A^{p+1}(p) \lambda^{\frac{2}{3}}-(1-p) M A^{p+1}(p) \lambda\right. \\
\left.+(1-p) \theta_{p} A(p) \lambda+O\left(\lambda^{\frac{4}{3}}\right)\right) .
\end{gathered}
$$

It is easy to verify that for $\lambda>0$ sufficiently small, the function $k(z) \triangleq$ $\left(z-\theta_{p}\right)+\lambda z(1-z) /(1+\lambda z)$ attains its maximum over $\left(-1 / \lambda, \zeta_{1}^{+}\right]$at $\zeta_{1}^{+}$and $k\left(\zeta_{1}^{+}\right)<0$. Therefore

$$
k^{2}(z) \geq k^{2}\left(\zeta_{1}^{+}\right)=\left(\delta_{1}^{+}\right)^{2}+\delta_{1}^{+} O(\lambda)=\frac{1}{4} \nu^{2} \lambda^{\frac{2}{3}}-\frac{1}{2} \nu^{2} \xi \lambda+o(\lambda),-\frac{1}{\lambda}<z \leq \zeta_{1}^{+} .
$$

It follows that for sufficiently small $\lambda>0$

$$
\begin{aligned}
\mathcal{D} w_{+} & (z)-\widetilde{U}\left((1-p) w_{+}(z)-z w_{+}^{\prime}(z)\right) \\
=\{ & \frac{1}{2} p \sigma^{2}\left(\left(z-\theta_{p}\right)+z(1-z) \frac{\lambda}{1+\lambda z}\right)^{2}-\gamma_{2} A^{p+1}(p) \lambda^{\frac{2}{3}}+M A^{p+1}(p) \lambda \\
& \left.\quad-\theta_{p} A(p) \lambda+O\left(\lambda^{\frac{4}{3}}\right)\right\}(1-p) w_{+}(z)
\end{aligned}
$$




$$
\begin{aligned}
\geq & \left\{\frac{1}{2} p \sigma^{2} k\left(\zeta_{1}^{+}\right)-\gamma_{2} A^{p+1}(p) \lambda^{\frac{2}{3}}+M A^{p+1}(p) \lambda-\theta_{p} A(p) \lambda+O\left(\lambda^{\frac{4}{3}}\right)\right\} \\
& \times(1-p) w_{+}(z) \\
= & \left\{\left(\frac{1}{8} p \sigma^{2} \nu^{2}-\gamma_{2} A^{p+1}(p)\right) \lambda^{\frac{2}{3}}+\left(M A^{p+1}(p)-\frac{1}{4} p \sigma^{2} \nu^{2} \xi-\theta_{p} A(p)\right) \lambda\right. \\
& +o(\lambda)\}(1-p) w_{+}(z) \\
=\{ & \left.\left(M A^{p+1}(p)-\frac{1}{4} p \sigma^{2} \nu^{2} \xi-\theta_{p} A(p)\right) \lambda+o(\lambda)\right\}(1-p) w_{+}(z) \geq 0,
\end{aligned}
$$

where we have used (4.13) in the last step.

Step $4 b$ : Interval $\left[\zeta_{2}^{+}, 1 / \lambda\right)$. This is analogous to Step $4 a$.

Step 4c: Interval $\left(\zeta_{1}^{+}, \zeta_{2}^{+}\right)$. From (4.19) and (4.13) we have

$$
\mathcal{D} w_{+}(z)-\widetilde{U}\left((1-p) w_{+}(z)-z w_{+}(z)\right)=\left(\theta_{p} A^{-p}(p)+M\right) \lambda+O\left(\lambda^{\frac{4}{3}}\right) \geq 0 .
$$

We must also show that

$$
g_{1}(z) \triangleq \lambda(1-p) w_{+}(z)-(1+\lambda z) w_{+}^{\prime}(z) \geq 0, \quad \zeta_{1}^{+}<z<\zeta_{2}^{+} .
$$

For $z \in\left(\zeta_{1}^{+}, \zeta_{2}^{+}\right)$, we have $z-\theta_{p}=O\left(\lambda^{1 / 3}\right)$. Using this fact, we compute

$$
\begin{aligned}
g_{1}(z)= & A^{-p}(p) \lambda-(1-p) \gamma_{2} \lambda^{\frac{5}{3}}+(1-p) M \lambda^{2} \\
& \quad-\frac{3(1-p)}{2 \nu} A^{-p}(p)\left(z-\theta_{p}\right)^{2} \lambda^{\frac{5}{3}}+\frac{1-p}{\nu^{3}} A^{-p}(p)\left(z-\theta_{p}\right)^{4} \lambda \\
& +\frac{3(1+\lambda z)}{\nu} A^{-p}(p)\left(z-\theta_{p}\right) \lambda^{\frac{2}{3}}-\frac{4(1+\lambda z)}{\nu^{3}} A^{-p}(p)\left(z-\theta_{p}\right)^{3}, \\
g_{1}^{\prime}(z)= & \frac{12}{\nu} A^{-p}(p) \lambda^{\frac{2}{3}}\left[\frac{1}{4}-\left(\frac{z-\theta_{p}}{\nu \lambda^{\frac{1}{3}}}\right)^{2}+O(\lambda)\right] .
\end{aligned}
$$

We know that $g_{1}\left(\zeta_{1}^{+}\right)=0$ and thus, to prove (4.22), it suffices to show that $g_{1}^{\prime}$ is positive on $\left[\zeta_{1}^{+}, \zeta_{2}^{+}\right]$. Because $-\left(z-\theta_{p}\right)^{2}$ is a concave function of $z$, it suffices to check the endpoints. We have for $i=1,2$ that

$$
\left(\frac{\zeta_{i}^{+}-\theta_{p}}{\nu \lambda^{\frac{1}{3}}}\right)^{2}=\left(\frac{1}{2}\left(1-\xi \lambda^{\frac{1}{3}}\right)+o\left(\lambda^{\frac{1}{3}}\right)\right)^{2}=\frac{1}{4}-\frac{1}{2} \xi \lambda^{\frac{1}{3}}+o\left(\lambda^{\frac{1}{3}}\right) .
$$

Therefore,

$$
g_{1}^{\prime}\left(\zeta_{i}^{+}\right)=\frac{12}{\nu} A^{-p}(p) \lambda^{\frac{2}{3}}\left[\frac{1}{2} \xi \lambda^{\frac{1}{3}}+o\left(\lambda^{\frac{1}{3}}\right)\right]>0
$$

for sufficiently small $\lambda>0$. The proof that

$$
g_{2}(z)=\lambda(1-p) w_{+}(z)+(1-\lambda z) w_{+}^{\prime}(z)
$$


is positive for $z \in\left[\zeta_{1}^{+}, \zeta_{2}^{+}\right]$is analogous. This completes the proof that $\left(w_{+}, \zeta_{1}^{+}, \zeta_{2}^{+}\right)$ is a supersolution triple.

\section{Conclusion:}

We note that $w_{ \pm}\left(\theta_{p}\right)=\frac{1}{1-p} A^{-p}(p)-\gamma_{2} \lambda^{\frac{2}{3}} \pm M \lambda$, and so Lemma 4.2 implies

$$
\frac{1}{1-p} A^{-p}(p)-\gamma_{2} \lambda^{\frac{2}{3}}-M \lambda \leq u\left(\theta_{p}\right) \leq \frac{1}{1-p} A^{-p}(p)-\gamma_{2} \lambda^{\frac{2}{3}}+M \lambda .
$$

Corollary 4.4. Assume $p>0, p \neq 1$ and $A(p)>0$. For fixed $z \in \mathcal{T}$, the value function satisfies

$$
u(z)=\frac{1}{1-p} A^{-p}(p)-\left(\frac{9}{32} p \theta_{p}^{4}\left(1-\theta_{p}\right)^{4}\right)^{\frac{1}{3}} A^{-1-p}(p) \sigma^{2} \lambda^{\frac{2}{3}}+O(\lambda) .
$$

Proof. In the proof of Theorem 4.3 we constructed a supersolution $w_{+}$and a subsolution $w_{-}$such that $w_{+}(z)-w_{-}(z)=O(\lambda), w_{ \pm}(z)=w_{ \pm}(\theta)+O(\lambda)$ for fixed $z \in \mathcal{T}$. It follows that $u(z)=w_{ \pm}(z)+O(\lambda)=w_{ \pm}(\theta)+O(\lambda)=u(\theta)+O(\lambda)$.

Theorem 4.5. Assume $p>0, p \neq 1, A(p)>0$ and $\theta_{p} \neq 1$. Then with $\nu$ given by (3.12), we have

$$
z_{1}=\theta_{p}-\frac{1}{2} \nu \lambda^{\frac{1}{3}}+O\left(\lambda^{\frac{2}{3}}\right), \quad z_{2}=\theta_{p}+\frac{1}{2} \nu \lambda^{\frac{1}{3}}+O\left(\lambda^{\frac{2}{3}}\right) .
$$

Proof. The value function $u$ is concave, so $u^{\prime}$ is monotone. By taking the derivative of $u$ in the $B S$ and $S S$ regions (see (2.12) and (2.13)) we see that

$$
u^{\prime}(z)=O(\lambda) \quad \forall z \in\left[z_{1}, z_{2}\right] .
$$

It follows that for $z=z_{1}$ and $z=z_{2}$, and hence for all $z \in\left[z_{1}, z_{2}\right]$,

$$
\widetilde{U}\left((1-p) u(z)-z u^{\prime}(z)\right)=\frac{p}{1-p} A^{1-p}(p)+(1-p) \gamma_{2} A(p) \lambda^{\frac{2}{3}}+O(\lambda) .
$$

We also know that $u^{\prime \prime}(z)$ is continuous for $z \in \mathcal{T} \backslash\{1\}$. From equations $(2.12)$ and (2.13) it follows that $u^{\prime \prime}\left(z_{i}\right)=O\left(\lambda^{2}\right)$ for $z_{i} \neq 1$. We can thus write

$$
\begin{aligned}
\mathcal{D} u\left(z_{i}\right) & =\left(p A(p)+\frac{1}{2} \sigma^{2} p(1-p)\left(z_{i}-\theta_{p}\right)^{2}\right) u\left(z_{i}\right)+O(\lambda) \\
& =\frac{p}{1-p} A^{1-p}(p)-p \gamma_{2} A(p) \lambda^{\frac{2}{3}}+\frac{1}{2} \sigma^{2} p A^{-p}(p)\left(z_{i}-\theta_{p}\right)^{2}+O(\lambda) .
\end{aligned}
$$

If $z_{i}=1$, the term $\left(1-z_{i}\right)^{2} u^{\prime \prime}\left(z_{i}\right)$ is set equal to zero (see [43], equation (A.5)), and the above equation still holds. In order to satisfy Equation (2.8) we must have

$$
\begin{aligned}
0 & =\mathcal{D} u\left(z_{i}\right)-\widetilde{U}\left((1-p) u\left(z_{i}\right)-z_{i} u^{\prime}\left(z_{i}\right)\right) \\
& =-\gamma_{2} A(p) \lambda^{\frac{2}{3}}+\frac{1}{2} \sigma^{2} p A^{-p}(p)\left(z_{i}-\theta_{p}\right)^{2}+O(\lambda) .
\end{aligned}
$$


It follows that

$z_{i}=\theta_{p} \pm \lambda^{\frac{1}{3}} \sqrt{\frac{2}{p \sigma^{2}} A^{p+1}(p) \gamma_{2}}+O\left(\lambda^{\frac{2}{3}}\right)=\theta_{p} \pm\left(\frac{3}{2 p} \theta_{p}^{2}\left(1-\theta_{p}\right)^{2}\right)^{\frac{1}{3}} \lambda^{\frac{1}{3}}+O\left(\lambda^{\frac{2}{3}}\right)$,

where the $-\operatorname{sign}$ is for $z_{1}$ and the $+\operatorname{sign}$ is for $z_{2}$.

Remark 4.6. The proof of Theorem 4.3 is valid so long as $\theta_{p} \neq 1$. The case of $\theta_{p}=1$ can be considered a singular case for which the parameter $\nu$ appearing in a denominator in the definition of $h(\delta)$ is zero. The intuition is that if the optimal proportion in the risky asset is $100 \%$ of wealth, then the investor does not incur any transaction costs due to adjusting the position size, as soon as the agent's position equals the optimum of $100 \%$ in stock. However, in order to consume the agent must transfer money from stock to money market and pay the transaction cost. We could regard this transaction cost as a consumption tax. It follows that the loss in value function is of order of $\lambda$ rather than $\lambda^{2 / 3}$. In [43], Theorems 11.2 and 11.6 show that in this case $z_{2}=\theta_{p}=1>z_{1}>0$, and [43], Corollary 9.10 asserts that

$$
v(x, y)=\frac{1}{1-p} A^{-p}(p)(x+(1-\lambda) y)^{1-p}, \quad(x, y) \in \mathcal{S}, x \leq 0,
$$

or equivalently,

$$
u(z)=v(1-z, z)=\frac{1}{1-p} A^{-p}(p)(1-\lambda z)^{1-p}, \quad 1 \leq z<\frac{1}{\lambda} .
$$

We see then that

$$
u\left(\theta_{p}\right)=\frac{1}{1-p} A^{-p}(p)-A^{-p}(p) \lambda+O\left(\lambda^{2}\right),
$$

which is consistent with (4.12).

The proof of Theorem 4.5 is valid for $\theta_{p}=1$, except the last step. When $\theta_{p}=1$, we have $\gamma_{2}=0$ and (4.25) reduces to

$$
z_{1}-\theta_{p}=z_{1}-1=O\left(\lambda^{\frac{1}{2}}\right) .
$$

Remark 4.7. The optimal consumption policy $c$ is given by $v_{x}^{-1 / p}$ (see (2.4) and (2.3)). Since $v(x, y)=(x+y)^{1-p} u(y /(x+y))$ (see (2.7)), we have

$$
\begin{aligned}
v_{x}(x, y) & =(1-p)(x+y)^{-p} u\left(\frac{y}{x+y}\right)-y(x+y)^{-1-p} u^{\prime}\left(\frac{y}{x+y}\right) \\
& =(1-p)(x+y)^{-p} u\left(\frac{y}{x+y}\right)+O(\lambda),
\end{aligned}
$$

where we have used (4.24). Therefore, Theorem 4.3 implies that the optimal consumption in feedback form is

$c=\left(A^{-p}(p)-(1-p) \gamma_{2} \lambda^{\frac{2}{3}}\right)^{-\frac{1}{p}}(x+y)+O(\lambda)=\left(A(p)+\frac{1-p}{p} \gamma_{2} \lambda^{\frac{2}{3}}\right)(x+y)+O(\lambda)$. 
Observe that for sufficiently small $\lambda$ the existence of transaction costs increases the size of consumption for $p \in(0,1)$, while the consumption is decreased for $p>1$. The intuition explaining this observation is related to the fact that the index of intertemporal substitution, which is equal to $1 / p$, is high for small $p$. An agent with index of intertemporal substitution higher than 1 will optimally avoid some transaction costs resulting from trading by consuming faster.

Remark 4.8. There is considerable evidence that

$$
u\left(\theta_{p}\right)=\frac{1}{1-p} A^{-p}(p)-\gamma_{2} \lambda^{\frac{2}{3}}-\theta_{p} A^{-p}(p) \lambda+O\left(\lambda^{\frac{4}{3}}\right) .
$$

We have just seen in (4.26) that this is the case when $\theta_{p}=1$ (and consequently $\left.\gamma_{2}=0\right)$. In the proof of Theorem 4.3, any choice of $M>\theta_{p} A^{-p}(p)$ gives us a subsolution of the form

$$
w_{-}\left(\theta_{p}\right)=\frac{1}{1-p} A^{-p}(p)-\gamma_{2} \lambda^{\frac{2}{3}}-M \lambda+O\left(\lambda^{\frac{4}{3}}\right) .
$$

(The second term on the right-hand side of (4.13) is needed only for the supersolution argument.) Finally, the coefficient $-\theta_{p} A^{-p}(p)$ on $\lambda$ can be obtained by a tedious heuristic analysis along the lines of Section 3.

In Remark 4.6 we introduced the concept of consumption tax as an interpretation of the transaction cost an agent must pay in order to first move capital from stock to money market before consuming it. In that remark, the agent was ideally $100 \%$ invested in stock. If instead the agent seeks to hold a proportion $\theta_{p} \neq 1$ in stock, then consuming proportionally from stock and money market, the agent would pay a consumption $\operatorname{tax} \lambda \theta_{p}$ times the total consumption. To highest order, the optimal consumption level is thus $\left(1-\lambda \theta_{p}\right)$ of what it would be if there were no transaction cost, and this multiplies the value function by

$$
\left(1-\lambda \theta_{p}\right)^{1-p}=1-(1-p) \theta_{p} \lambda+O\left(\lambda^{2}\right) .
$$

The value function for zero transaction cost when wealth is 1 is $A^{-p}(p) /(1-p)$, and so after this multiplication, the value function has been reduced by

$$
\theta_{p} A^{-p}(p) \lambda,
$$

which is the order $\lambda$ term we see in (4.27). We thus expect the value function for the problem with transaction cost $\lambda$ to be reduced from the zero-transaction cost value function $A^{-p}(p) /(1-p)$ by at least $\theta_{p} A^{-p}(p) \lambda$, this reduction being due solely to the cost of moving capital from stock to money market in order to consume. There is also a cost of trading to stay in the $N T$ wedge, which reduces the value function by $\gamma_{2} \lambda^{\frac{2}{3}}$, but cannot further reduce the value function by an order $\lambda$ term because then the value function would fall below the lower bound (4.28).

Under the assumption that (4.27) holds, one can improve the calculations in Theorem 4.5. We already have from Theorem 4.5 and its proof that $z_{i}-\theta_{p}=$ $O\left(\lambda^{1 / 3}\right)$ and that $u^{\prime}(z)=O(\lambda)$ for $z \in\left[z_{1}, z_{2}\right]$. The mean-value theorem gives

$$
u\left(z_{i}\right)=u\left(\theta_{p}\right)+O\left(\lambda^{\frac{4}{3}}\right),
$$


and

$$
\begin{aligned}
(1-p) u\left(z_{1}\right)-z_{1} u^{\prime}\left(z_{1}\right) & =\frac{(1-p) u\left(z_{1}\right)}{1+\lambda z_{1}} \\
& =A^{-p}(p)-(1-p) \gamma_{2} \lambda^{\frac{2}{3}}-(2-p) \theta_{p} A^{-p}(p) \lambda+O\left(\lambda^{\frac{4}{3}}\right), \\
(1-p) u\left(z_{2}\right)-z_{2} u^{\prime}\left(z_{2}\right) & =\frac{(1-p) u\left(z_{2}\right)}{1-\lambda z_{2}} \\
& =A^{-p}(p)-(1-p) \gamma_{2} \lambda^{\frac{2}{3}}+p \theta_{p} A^{-p}(p) \lambda+O\left(\lambda^{\frac{4}{3}}\right) .
\end{aligned}
$$

Using Lemma 2.1, we obtain

$$
\begin{aligned}
& \widetilde{U}\left((1-p) u\left(z_{2}\right)-z_{2} u^{\prime}\left(z_{2}\right)\right) \\
& \quad=\frac{p}{1-p} A^{1-p}(p)+(1-p) A(p) \gamma_{2} \lambda^{\frac{2}{3}}+(2-p) \theta_{p} A^{1-p}(p) \lambda+O\left(\lambda^{\frac{4}{3}}\right), \\
& \widetilde{U}\left((1-p) u\left(z_{1}\right)-z_{1} u^{\prime}\left(z_{1}\right)\right) \\
& \quad=\frac{p}{1-p} A^{1-p}(p)+(1-p) A(p) \gamma_{2} \lambda^{\frac{2}{3}}-p \theta_{p} A^{1-p}(p) \lambda+O\left(\lambda^{\frac{4}{3}}\right) .
\end{aligned}
$$

We write for $z_{1}$ and $z_{2}$ similarly as in Theorem 4.5

$\mathcal{D} u\left(z_{i}\right)=\frac{p}{1-p} A^{1-p}(p)-p A(p) \gamma_{2} \lambda^{\frac{2}{3}}-p \theta_{p} A^{1-p}(p) \lambda+\frac{1}{2} \sigma^{2} p A^{-p}(p)\left(z_{i}-\theta_{p}\right)^{2}+O\left(\lambda^{\frac{4}{3}}\right)$.

From $\mathcal{D} u\left(z_{i}\right)=\widetilde{U}\left((1-p) u\left(z_{i}\right)-z_{i} u^{\prime}\left(z_{i}\right)\right)$, we now see that

$$
\begin{aligned}
\left(z_{1}-\theta_{p}\right)^{2} & =\frac{2}{p \sigma^{2}}\left(A^{1+p}(p) \gamma_{2} \lambda^{\frac{2}{3}}+2 \theta_{p} A(p) \lambda\right)+O\left(\lambda^{\frac{4}{3}}\right), \\
\left(z_{2}-\theta_{p}\right)^{2} & =\frac{2}{p \sigma^{2}} A^{1+p}(p) \gamma_{2} \lambda^{\frac{2}{3}}+O\left(\lambda^{\frac{4}{3}}\right),
\end{aligned}
$$

which implies

$$
\begin{aligned}
& z_{1}-\theta_{p}=-\frac{1}{2} \nu \lambda^{\frac{1}{3}}-\frac{4 \theta_{p} A(p)}{\sigma^{2} p \nu} \lambda^{\frac{2}{3}}+O(\lambda), \\
& z_{2}-\theta_{p}=\frac{1}{2} \nu \lambda^{\frac{1}{3}}+O(\lambda) .
\end{aligned}
$$

This suggests that the optimal policy is to keep a wider wedge on the right side of the Merton proportion $\theta_{p}$. This extra width makes sense because consumption reduces the money market position.

We can do the same calculation for $\theta_{p}=1$, in which case $\gamma_{2}=\nu=0$. Taking the square roots in (4.29), (4.30), we now have

$$
z_{1}-\theta_{p}=z_{1}-1=-\sqrt{\frac{4 \theta_{p} A(p)}{\sigma^{2} p}} \lambda^{\frac{1}{2}}+O\left(\lambda^{\frac{5}{6}}\right), \quad z_{2}-\theta_{p}=z_{2}-1=O(\lambda) .
$$

In fact, when $\theta_{p}=1$, we have $z_{2}=\theta_{p}$. 
Remark 4.9. In the key formulas derived in this paper, the transaction cost parameter $\lambda$ appears in combination with $\Gamma \triangleq \theta_{p}\left(1-\theta_{p}\right)$. According to Theorem 4.3 , the highest order loss in the value function due to transaction costs is

$$
\left(\frac{9 p}{32}\right)^{\frac{1}{3}} A^{-1-p} \sigma^{2}\left(\Gamma^{2} \lambda\right)^{\frac{2}{3}}
$$

From Theorem 4.5, we see that

$$
z_{i}-\theta_{p}=(-1)^{i}\left(\frac{3}{2 p}\right)^{\frac{1}{3}}\left(\Gamma^{2} \lambda\right)^{\frac{1}{3}} .
$$

One way to see the intrinsic nature of the quantity $\Gamma^{2} \lambda$ is to define the proportion of capital in stock, $\theta_{t}=Y_{t} /\left(X_{t}+Y_{t}\right)$, and apply Itô's formula when $\left(X_{t}, Y_{t}\right)$ is generated by the optimal triple $(C, L, M)$, for which $L$ and $M$ are continuous, to derive the equation

$$
\begin{gathered}
d \theta_{t}=\theta_{t}\left(1-\theta_{t}\right) \frac{d S_{t}}{S_{t}}-r \theta_{t}\left(1-\theta_{t}\right) \mathrm{d} t-\sigma^{2} \theta_{t}^{2}\left(1-\theta_{t}\right) \mathrm{d} t+\frac{\theta_{t} C_{t}}{X_{t}+Y_{t}} \mathrm{~d} t \\
+\frac{\lambda \theta_{t}+1}{X_{t}+Y_{t}} d L_{t}+\frac{\lambda \theta_{t}-1}{X_{t}+Y_{t}} d M_{t} .
\end{gathered}
$$

We see that the response of $\theta_{t}$ to relative changes in the stock price is $\theta_{t}\left(1-\theta_{t}\right)$. Our desire is to keep $\theta_{t}$ in the interval $\left[z_{1}, z_{2}\right]$. If the leading term in the above equation were scaled to be $\rho \theta_{t}\left(1-\theta_{t}\right) \frac{d S_{t}}{S_{t}}$, then because the $\mathrm{d} t$ terms have a lower order effect on the dynamics, this would be approximately equivalent to scaling time by $\rho^{2}$, and so the local time of $\theta_{t}$ at the endpoints of $\left[z_{1}, z_{2}\right]$ would approximately be scaled by $\rho^{2}$. If at the same time the transaction cost were replaced by $\lambda / \rho^{2}$, then the total amount of transacting would be approximately unaffected. The quantity $\theta_{t}^{2}\left(1-\theta_{t}\right)^{2} \lambda$ is invariant under this scaling. Because the optimal policy keeps $\theta_{t}$ near $\theta_{p}$, the quantity $\Gamma^{2} \lambda$ appears to be intrinsic.

When replicating an option by trading, the position held by the hedging portfolio, denominated in shares of stock, is call the delta of the option, and the sensitivity of the delta to changes in the stock price is the gamma. We have here a similar situation, except that $\theta_{t}$ is the proportion of capital held in stock, rather than the number of shares of stock, and $\theta_{t}\left(1-\theta_{t}\right)$ is the sensitivity of this proportion to relative changes in the stock price.

It is interesting to note that the quantity $\Gamma^{2} \lambda$ also plays a fundamental role in the formal asymptotic expansions of Whalley and Wilmott [49]. In fact, even the constants $\left(\frac{9}{32}\right)^{\frac{1}{3}}$ and $\left(\frac{3}{2}\right)^{\frac{1}{3}}$ in (4.31) and (4.32) appear in [49], the first at the end of Section 3.3 and the second in equation (3.10). 


\section{A Width of the $N T$ interval}

We shall only consider $\delta$ of the form $O\left(\lambda^{1 / 3}\right)$. For such $\delta$, we may write the terms of order $\lambda$ and lower in $f_{1}^{ \pm}(\delta)$ of (4.14) as

$$
\begin{aligned}
& f_{1}^{ \pm}(\delta) \\
& \quad=\nu \lambda^{\frac{1}{3}}-(1-p) \gamma_{2} \nu A^{p}(p) \lambda-h^{\prime}(\delta) \lambda^{-\frac{2}{3}}-\theta_{p} h^{\prime}(\delta) \lambda^{\frac{1}{3}}+h^{\prime}(\delta) \delta \lambda^{\frac{1}{3}}+O\left(\lambda^{\frac{4}{3}}\right) \\
& \quad=\nu \lambda^{\frac{1}{3}}-(1-p) \gamma_{2} \nu A^{p}(p) \lambda-3 \delta+\frac{4}{\nu^{2}} \cdot \frac{\delta^{3}}{\lambda^{\frac{2}{3}}}-3 B \delta \lambda^{\frac{2}{3}}+O\left(\lambda^{\frac{4}{3}}\right) .
\end{aligned}
$$

Consider $\delta_{0} \triangleq \frac{1}{2} \nu \lambda^{1 / 3}\left(1-\xi_{0} \lambda^{1 / 3}\right)$. Then

$$
\begin{aligned}
f_{1}^{ \pm}\left(\delta_{0}\right)= & \nu \lambda^{\frac{1}{3}}-(1-p) \gamma_{2} \nu A^{p}(p) \lambda-\frac{3}{2} \nu \lambda^{\frac{1}{3}}+\frac{3}{2} \nu \xi_{0} \lambda^{\frac{2}{3}} \\
& +\frac{1}{2} \nu \lambda^{\frac{1}{3}}\left(1-3 \xi_{0} \lambda^{\frac{1}{3}}+3 \xi_{0}^{2} \lambda^{\frac{2}{3}}\right)-\frac{3}{2} B \nu \lambda+O\left(\lambda^{\frac{4}{3}}\right) \\
= & \nu\left(\frac{3}{2} \xi_{0}^{2}-(1-p) \gamma_{2} A^{p}(p)-\frac{3}{2} B\right) \lambda+O\left(\lambda^{\frac{4}{3}}\right) .
\end{aligned}
$$

With $\xi=\sqrt{\frac{2}{3}(1-p) \gamma_{2} A^{p}(p)+B}>0$, we take $\xi_{0}=\sqrt{\xi^{2}+\eta}$, where $|\eta|<$ $\xi^{2}$. Then $f_{1}^{ \pm}\left(\delta_{0}\right)=\frac{3}{2} \nu \eta \lambda+O\left(\lambda^{\frac{4}{3}}\right)$. Thus, for $\eta>0$ we have $f_{1}^{ \pm}\left(\delta_{0}\right)>0$ for sufficiently small $\lambda>0$, and for $\eta<0$ we have $f_{1}^{ \pm}\left(\delta_{0}\right)<0$ for sufficiently small $\lambda>0$. Therefore, for every $\eta \in\left(0, \xi^{2}\right)$ and sufficiently small $\lambda>0$, there exists

$$
\delta_{1}^{ \pm} \in\left(\frac{1}{2} \nu \lambda^{\frac{1}{3}}\left(1-\lambda^{\frac{1}{3}} \sqrt{\xi^{2}-\eta}\right), \frac{1}{2} \nu \lambda^{\frac{1}{3}}\left(1-\lambda^{\frac{1}{3}} \sqrt{\xi^{2}+\eta}\right)\right)
$$

satisfying $f_{1}^{ \pm}\left(\delta_{1}^{ \pm}\right)=0$. In other words, $\delta_{1}^{ \pm}=\frac{1}{2} \nu \lambda^{\frac{1}{3}}\left(1-\xi \lambda^{\frac{1}{3}}\right)+o\left(\lambda^{\frac{2}{3}}\right)$. The proof of the existence of $\delta_{2}^{ \pm}$is analogous.

\section{References}

[1] Akian, M., Menaldi, J. L. \& Sulem, A., On an investment consumption model with transaction costs, SIAM J. Control 34, 329-364 (1996).

[2] Atkinson, C. \& Wilmott, P., Portfolio management with transaction costs: an asymptotic analysis of the Morton and Pliska model, Math. Finance 5, 357-367 (1995).

[3] Avellaneda, M. \& Parás, A., Dynamic hedging portfolios for derivative securities in the presence of large transaction costs, Appl. Math. Finance 1, 165-194 (1994).

[4] Barles, G. \& Soner, H. M., Option pricing with transaction costs and a nonlinear Black-Scholes equation, Finance Stoch. 2, 369-397 (1998). 
[5] Bensaid, B., Lesne, J. P., Pagés, H. \& Scheinkman, J., Derivative asset pricing with transaction costs, Math. Finance 2, 63-86 (1992).

[6] Bouchard-Denize, B. \& Touzi, N., Explicit solution of the multivariable super-replication problem under transaction costs, Annals of Applied Probability 10, 685-708 (2000).

[7] Boyle, P. P. \& Vorst, T., Option replication in discrete time with transaction costs, J. Finance 47, 272-293 (1992).

[8] Constantinides, G., Multiperiod consumption and investment behavior with convex transaction costs, Management Sci. 25, 1127-1137 (1979).

[9] Constantinides, G., Capital market equilibrium with transaction costs, J. Political Economy 94, 842-862 (1986).

[10] Constantinides, G. \& Zariphopoulou, Th., Bounds on prices of contingent claims in an intertemporal economy with proportional transaction costs and general preferences, Finance Stoch. 3, 345-369 (1999).

[11] Constantinides, G. \& Zariphopoulou, Th., Bounds on derivative prices in an intertemporal setting with proportional transaction costs and multiple securities, Math. Finance 11, 331-346 (2001).

[12] Cvitanič, J. \& Karatzas, I., Hedging and portfolio optimization under transaction costs, Math. Finance 6, 113-165 (1996).

[13] Cvitanič, J. \& Wang, H., On optimal terminal wealth under transaction costs, J. Mathematical Economics 35, 223-232. (2001)

[14] Cvitanič, J., Pham, H. \& Touzi, N, A closed-form solution to the problem of super-replicating under transaction costs, Finance Stoch. 3, 35-54 (1999).

[15] Davis, M. H. A. \& Norman, A., Portfolio selection with transaction costs, Math. Oper. Res. 15, 676-713 (1990).

[16] Davis, M. H. A., Panas, V. G. \& Zariphopoulou, Th., European option pricing with transaction costs, SIAM J. Control 31, 470-493 (1993).

[17] Deelstra, G., Pham, H. \& Touzi, N., Dual formulation of the utility maximization problem under transaction costs, Annals of Applied Probability 11, 1353-1383 (2001).

[18] Delbaen, F., Kabanov, Yu. \& Valkeila, E., Hedging under transaction costs in currency markets: a discrete-time model, Math. Finance 12, 45-61 (2002).

[19] Edirisinghe, C., Naik, V. \& Uppal, R., Optimal replication of options with transaction costs and trading restrictions, J. Finan. Quant. Anal. 28, 117-138 (1993). 
[20] Figlewski, S., Options arbitrage in imperfect markets, J. Finance 44, 1289-1311 (1989).

[21] Framstad, N., Øksendal, B. \& Sulem, A., Optimal consumption and portfolio in a jump-diffusion market with proportional transaction costs, J. Math. Econ. 35, 233-257 (2001).

[22] Gilster, J. \& Lee, W., The effects of transaction costs and different borrowing and lending rates on the option pricing problem: A note, $J$. Finance 39, 1215-1222 (1984).

[23] Grannan, E. R. \& Swindle, G., Minimizing transaction costs of option hedging strategies, Math. Finance 6, 341-364 (1996).

[24] Hodges, S. \& Neuberger, A., Option replication of contingent claims under transaction costs, Rev. Futures Markets 8, 222-239 (1989).

[25] Hoggard, T., Whalley, A. \& Wilmott, P. , Hedging option portfolios in the presence of transaction costs, Adv. Fut. Oper. Res. 7, 21-35 (1994).

[26] Jouni, E. , Market imperfections, equilibrium, and arbitrage, in Lecture Notes in Mathematics 1656, Springer-Verlag, Berlin, pp. 247-307 (1997).

[27] Jouni, E. \& Kallal, H., Martingales and arbitrage in securities markets with transaction costs, J. Econ. Theory 66, 178-197 (1995).

[28] Kabanov, Yu., Hedging and liquidation under transaction costs, Finance Stoch. 3, 237-348 (1999).

[29] Kabanov, Yu. \& Klüppelberg, C. , A geometric approach to portfolio optimization in models with transaction costs, Submitted for publication.

[30] Kabanov, Yu. \& Last, G. , Hedging under transaction costs in currency markets, Math. Finance 12, 63-70 (2002).

[31] Kabanov, Yu. \& Stricker, C. Hedging of contingent claims under transaction costs. In: Sandman, K., Schönbucher, P. (eds.) Advances in Finance and Stochastics. Essays in Honour of Dieter Sondermann. Berlin Heidelberg New York: Springer 2002.

[32] Karatzas, I. \& Shreve, S. Brownian Motion and Stochastic Calculus, Springer-Verlag, New York, 1988.

[33] Koenl, P. F., Pham, H. \& Touzi, N., Hedging in discrete-time under transaction costs and continuous-time limit, J. Applied Probability 36, 163-178 (1999).

[34] Koenl, P. F., Pham, H. \& Touzi, N., On super-replication under transaction costs in general discrete-time models, Theory of Probability and Applications 45, 783-788 (1999). 
[35] Kusuoka, S., Limit theorem option replication with transaction costs, Ann. Appl. Probab. 5, 198-221 (1995).

[36] Leland, H., Option pricing and replication with transaction costs, $J$. Finance 40, 1283-1301 (1985).

[37] Leventhal, S. \& Skorohod, A., On the possibility of hedging options in the presence of transaction costs, Ann. Appl. Probab. 7, 410-443 (1997).

[38] Loewenstein, M., On optimal portfolio trading strategies for an investor facing transaction costs in a continuous trading market, J. Math. Econ. 33, 209-228 (2000).

[39] Magill, M. J. .P. and Constantinides, G. M., Portfolio selection with transaction costs, J. Econ. Theory 13, 245-263 (1976).

[40] Merton, R., Optimum consumption and portfolio rules in a continuoustime case, J. Econ. Theory 3, 373-413 (1971) [Erratum 6, 213-214 (1973)].

[41] Morton, A. \& Pliska, S. R., Optimal portfolio management with fixed transaction costs, Math. Finance 5, 337-357 (1995).

[42] Samuelson, P. , Why we should not make mean log of wealth big though years to act are long, J. Banking Finance 3, 305-307 (1979).

[43] Shreve, S. \& Soner, H. M., Optimal investment and consumption with transaction costs, Ann. Applied Probab. 4, 609-692 (1994).

[44] Soner, H. M., Shreve, S. \& Cvitanič, J., There is no nontrivial hedging portfolio for option pricing with transaction costs, Ann. Appl. Probab. 5, 327-355 (1995).

[45] Tofт, K. B., On the mean-variance tradeoff in option replication with transaction costs, J. Financial Quant. Anal. 31, 233-263 (1996).

[46] Tourin, A. \& Zariphopoulou, Th., Numerical schemes for investment models with singular transactions, Computat. Econ. 7, 287-307 (1994).

[47] Tourin, A. \& Zariphopoulou, Th., Viscosity solutions and numerical schemes for investment/consumption models with transaction costs, in Numerical Methods in Finance, L. C. G. Rogers and D. Talay, eds., Isaac Newton Institute Publications (1997).

[48] TouzI, N., Super-replication under proportional transaction costs: from discrete-to-continuous time models, Math. Methods Oper. Res. 50, 297-320 (1999).

[49] Whalley, A. E. \& Wilmott, P., An asymptotic analysis of an optimal hedging model for option pricing under transaction costs, Math. Finance 7, 307-324 (1997). 